\title{
Tension-Induced Phase Transformation and Anomalous Poisson Effect in Violet Phosphorene
}

\author{
Penghua Ying ${ }^{\mathrm{a}}$, Xiaowen $\mathrm{Li}^{\mathrm{b}}$, Xiaobin Qiang ${ }^{\mathrm{b}}$, Yao Du ${ }^{\mathrm{a}}$, Jin Zhang*a ${ }^{* \mathrm{a}}$ Lang Chen ${ }^{\mathrm{b}}$, Zheng \\ Zhong ${ }^{\ddagger a}$ \\ ${ }^{a}$ School of Science, Harbin Institute of Technology, Shenzhen, 518055, PR China \\ ${ }^{b}$ Department of Physics, Southern University of Science and Technology, Shenzhen, 518055, PR China
}

\begin{abstract}
Two-dimensional violet phosphorene (VP) nanosheets are promising semiconductor materials with unique cross structures distinct from those of their allotropes such as black phosphorene and blue phosphorene, but their mechanical behaviors remain almost unexplored. By using the first-principles calculations, in this paper we investigate the mechanical behaviors of monolayer, bilayer, and bulk VP under uniaxial tension. A phase transformation from the open-pore phase to closed-pore phase is observed in VP structures when under a specific tensile strain. It is revealed that the phase transformation is attributed to the competition between the rotation and elongation of sub-nano rods in VP structures during the loading process. Due to the phase transformation, the in-plane Poissons ratio of monolayer VP can become greater than 1.2, while the bulk VP possesses a negative out-of-plane Poissons ratio with a magnitude up to -0.3 at a certain strain. These results indicate that Poisson effects in VP are superior to those in any other existing two-dimensional materials. In addition, based on the tensor analysis of elastic constants, a strong mechanical anisotropy is observed in VP structures both before and after the phase transformation. Besides the mechanical properties, the band gap of all VP structures decreases as the applied tensile strain increases, which can eventually transform into the metallic state prior to their fracture. The combination of unique phase transformation, anomalous Poisson effect, strong mechanical anisotropy and tunable electronic properties render VP be a novel nanoscale metamaterial with multifunctional applications.
\end{abstract}

Keywords: Violet phosphorene, Poisson's ratio, Phase transformation, Strain engineering, Mechanical anisotropy

\footnotetext{
*Corresponding author

$\dagger$ Corresponding author

$\ddagger$ Corresponding author

Email addresses: jinzhang@hit.edu.cn (Jin Zhang), chenlang@sustech.edu.cn (Lang Chen), zhongzheng@hit.edu.cn (Zheng Zhong)
} 


\section{Introduction}

Two-dimensional (2D) materials such as graphene and black phosphorene (BP) are attracting a great deal of attention due to their unique physical properties and the corresponding novel phenomena (Geim (2009); Carvalho et al. (2016)). Different to the zero band gap of semimetal graphene, $\mathrm{BP}$ is a semiconductor with the direct band gap ranging from $0.3 \mathrm{eV}$ (bulk) to $2.0 \mathrm{eV}$ (monolayer) (Tran et al. (2014)). Specifically, the high carrier mobility together with the high optical and ultraviolet absorption of BP makes it appealing for the use in next-generation optoelectronics (Carvalho et al. (2016)). However, the chemical instability such as fast oxidation and degradation of BP has been recognized as the bottleneck in its applications. Very recently, another allotrope of layered phosphorus named violet phosphorus (VP) was synthesized in experiments (Zhang et al. (2020c)), which was demonstrated to be a semiconductor with a direct band gap of $1.7 \mathrm{eV}$ (Zhang et al. (2020c)). Moreover, the VP having a unique 2D nanosized cross structure (Zhang et al. (2021)) was proven to be more thermally and chemically stable than its BP counterpart (Zhang et al. (2020c); Fali et al. (2021)), though BP was previously considered as the most stable phosphorus allotrope. In other words, VP possesses a much better thermal and chemical stability performance than its BP and, simultaneously, can retain the direct band gap property, which suggest that VP materials are more promising candidates for semiconductor-related applications (Zhang et al. (2020a)).

Understanding the mechanical properties of 2D semiconducting materials is not only crucial for the reliability of their applications in practical working environment but also of fundamental importance for the coupling between mechanical and other physical properties (Akinwande et al. (2017)). For example, the strain engineering technique has been extensively employed in previous studies to tune the electronic, optical, thermal, and piezo/flexoelectrical properties of 2D semiconducting materials (Dai et al. (2019)). Unlike its BP counterpart whose mechanical properties have been extensively investigated theoretically and experimentally (Jiang and Park (2014a); Wei and Peng (2014); Sha et al. (2015)), the investigation on mechanical properties of VP is currently very limited. Until very recently, the initial experimental attempt was conducted by Zhang et al. (2021) with the aid of atomic force microscope (AFM) based nanoindentation. The VP was demonstrated to possess an extremely high deformation resistance. The 2D Young's modulus of monolayer VP was reported to be $1512 \pm 76 \mathrm{Nm}^{-1}$, which is 4.4 times as high as that of graphene. However, restricted by the loading method of the AFM-based nanoindentation, the mechanical behavior of VP under in-plane uniaxial tension cannot be fully revealed in the existing experiments. Furthermore, owing to its unique cross structure at the nanoscale, it is expected that the VP may have a distinct mechanical behavior under in-plane loading, which may have a strong influence on its band gap property, similar to the strain engineering of band gaps of other 2D materials such as graphene (Lu and Guo (2010)), hexagonal BN (Wu et al. (2013)), $\mathrm{MoS}_{2}$ (Scalise et al. (2012)), and $\mathrm{Bi}_{2} \mathrm{O}_{2} \mathrm{Se}$ (Pang and $\mathrm{Li}(2021)$ ).

To this end, in the present work the first-principles calculations are performed to investigate the mechanical behaviors of monolayer, bilayer, and bulk VP under uniaxial tension. 
A tension-induced phase transformation phenomenon is observed in all VP structures. The thermodynamic and mechanical stability of the new phase is verified by molecular dynamics simulations and Born stability criteria. The mechanical properties of transformed new phase are compared with the values of its parent counterpart. Moreover, during the tension process of VP structures, an extremely large Poisson's ratio is found in the in-plane direction while a negative Poisson's ratio is found in the out-of-plane direction. This anomalous Poisson effect is explained by analysing the deformation mechanism of VP structures. In addition, the band gap of all VP structures is found to decrease with growing tensile strain, which eventually can transform into the metallic state at a large tensile strain. Our work not only reveals the potential of VP as nanoscale metamaterial with an extremely large in-plane Poisson's ratio and a negative out-of-plane Poisson's ratio from a fundamental perspective but also facilitates the applications of VP as a new class of band gap-tunable semiconductor derived by strain engineering.

\section{Model and methods}

The initial atomic structures of VP are shown in Fig. 1, the corresponding lattice parameters of which were extracted from the references (Zhang et al. (2020c,b)). The VP materials with different thickness, i.e., monolayer, bilayer, and bulk VP structures were considered in this work to investigate the effect of thickness on the mechanical behaviours of VP. The VP consisting of two layers has a orthogonal network structure (see Fig. 1(a)), in which each component layer is composed of cross sub-nano rods with -[P2]-[P8]-[P2]-[P9]repeating units and connected with the other layer through [P9] unit linking (see Fig. 1(c)). The side view of bulk VP considered in simulations is shown Fig. 1(b), while the simulation models of monolayer and bilayer VP can be further obtained by adding a sufficient vacuum layer of $15 \AA$ in the cross-plane plane ( $z$ direction).

All first-principles calculations were performed at the level of density functional theory (DFT), which were implemented through Vienna Ab initio Simulation Package (VASP) (Kresse and Furthmüller (1996))) with the aid of SCAN meta-GGA + rVV10 vdW functional form (Peng et al. (2016)). The calculations were converged with an energy tolerance of $10^{-5}$ $\mathrm{eV}$ and a force tolerance of $0.01 \mathrm{eV} / \AA$ under a cutoff energy of $500 \mathrm{eV}$. All computations were carried out by automatic $k$-mesh generators with $l=0.03$, where $l$ in the unit of $2 \pi / \AA$ is the $k$-points resolved value between adjacent $k$-points in reciprocal cell and. The number $N$ of $k$-points is further determined from

$$
N=\max (1,|\vec{b}| / l),
$$

where $\vec{b}$ is the reciprocal lattice vector in the specific direction. The optimized lattice parameters of monolayer, bilayer, and bulk VP are $8.89 \AA \times 9.00 \AA, 8.89 \AA \times 9.03 \AA$, and 8.92 $\AA \times 9.10 \AA \times 20.56 \AA$, respectively, which are comparable to the experimental results (Zhang et al. (2020c)). 
(a)

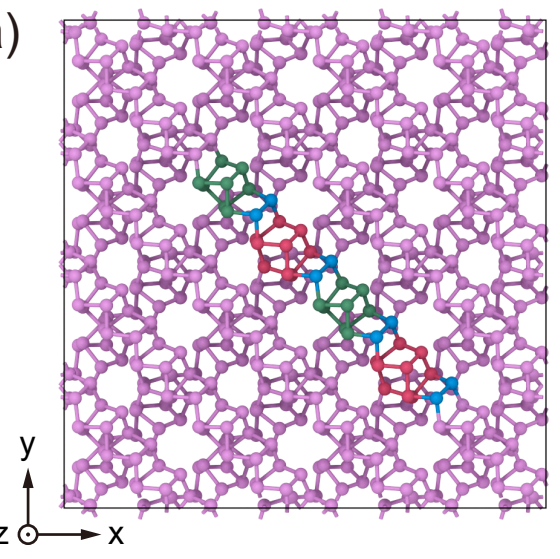

(b)

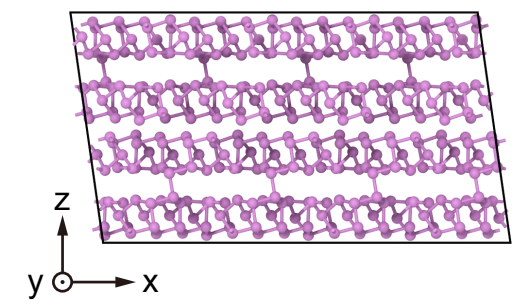

(c)

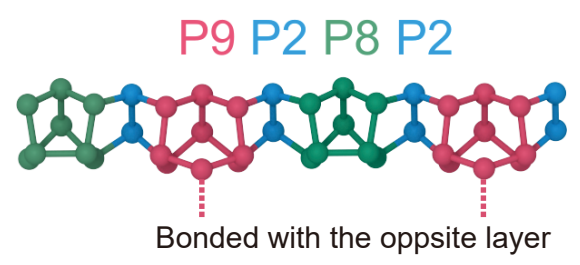

Figure 1: Atomic structures of VP considered in this work. (a) Top view of monolayer VP. (b) Side view of bulk VP. (c) The sub-nano rods of VP consisting of -[P2]-[P8]-[P2]-[P9]- repeating units. Here, the 4 $\times 4 \times 1$ supercells are presented for clarity. All atomic structures are drawn with aid of OVITO package (Stukowski (2009)).

\section{Results and discussions}

In this section, the mechanical responses, phase transformation, and electronic properties of VP structures obtained from DFT calculations are discussed. Efforts are made to further explain the mechanism of some observed novel phenomenon including anisotropic elastic properties, the extremely large in-plane Poisson's ratio, and the negative out-of-plane Poisson's ratio.

\subsection{Tension-Induced phase transformation}

We first investigated the mechanical behaviours of monolayer, bilayer, and bulk VP under uniaxial tension. Considering the similarity of the atomic structure of VP along $x$ and $y$ directions, the tension was applied only along the $x$ direction, while the stress along the lateral direction ( $y$ direction) was released to zero. Herein, we define the engineering strain as $\varepsilon_{i}=1-L_{i} / l_{i}$, where $L$ and $l$ denote the crystal lengths of the structure after and before deformation, respectively, and the subscripts $i=x, y$ and $z$ correspond to lengths in the $x, y$ and $z$ directions, respectively. The uniaxial tension was applied with a small strain increment of $1 \%$ each step. In this loading process, the corresponding stress was calculated as the total force divided by the cross-sectional area that is $L_{y} L_{z}$. The thickness $L_{z}$ of monolayer VP was set as a constant value of $10.28 \AA$, corresponding to the layer separation in bulk VP. For bilayer VP, $L_{z}$ was set as the sum of the distance of the furthest atom pairs $D$ and the closest atom pairs $d$ between upper and lower layers, i.e., $L_{z}=(D+d) / 2$ as illustrated in the inset of Fig. 2(d). The in-plane and out-of-plane Poisson's ratios, i.e., $v_{i n}$ and $v_{\text {out }}$, can be further obtained:

$$
\begin{aligned}
& v_{\text {in }}=-\frac{\varepsilon_{y}}{\varepsilon_{x}}, \\
& v_{\text {out }}=-\frac{\varepsilon_{z}}{\varepsilon_{x}} .
\end{aligned}
$$


Figure 2 shows the evolution of stress $\left(\sigma_{x}\right)$, strain energy $(U)$, and Poisson's ratios $\left(v_{i n}\right.$ and $\left.v_{\text {out }}\right)$ of VP structures with increasing strain $\left(\varepsilon_{x}\right)$ until fracture. From Fig. 2(a), it can be found that the whole tension process of VP mainly includes three stages. At the first stage, $\sigma_{x}$ increases linearly with growing $\varepsilon_{x}$ when $0<\varepsilon_{x} \lesssim 0.05$ and then keeps around a constant value at $0.05 \lesssim \varepsilon_{x} \lesssim 0.1$, which corresponds to the transformation from the elastic deformation to the plastic deformation. At the second stage, $\sigma_{x}$ keeps decreasing with growing $\varepsilon_{x}$ when $0.1 \lesssim \varepsilon_{x} \lesssim 0.2$ and finally reaches the minimum value at $\varepsilon_{x} \approx 0.2$, showing an abnormal negative-stiffness behavior at this stage. At the final stage $\left(\varepsilon_{x} \gtrsim 0.2\right)$, $\sigma_{x}$ turns to increase monotonically with increasing $\varepsilon_{x}$ until the final fracture of VP. As for three VP structures with different thicknesses considered here, the fracture strain is found to decrease with increasing thickness, while the tensile strength of different VP structures follows the order of monolayer $>$ bulk $>$ bilayer. Specifically, according to Figure 2(a), the tensile strength (fracture strain) of monolayer, bilayer, bulk VP is $14.15 \mathrm{GPa}(0.49), 11.79$ $\mathrm{GPa}(0.45)$, and $12.96 \mathrm{GPa}(0.42)$, respectively. Herein, the fracture strain is defined as the strain corresponding to the maximum stress, i.e., tensile strength.

(a)

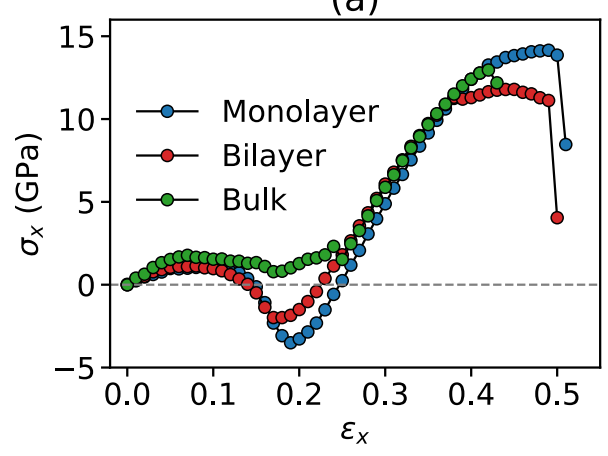

(c)

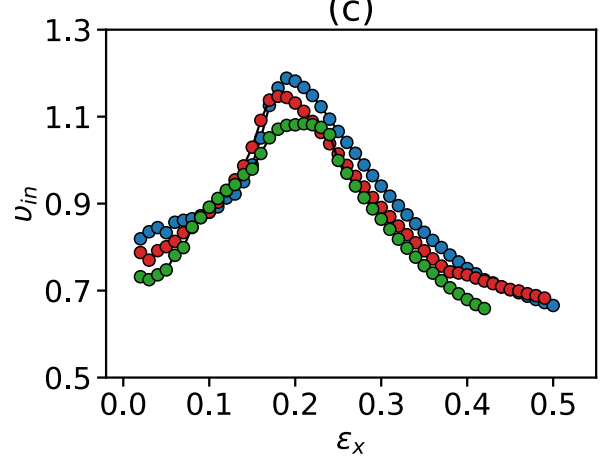

(b)

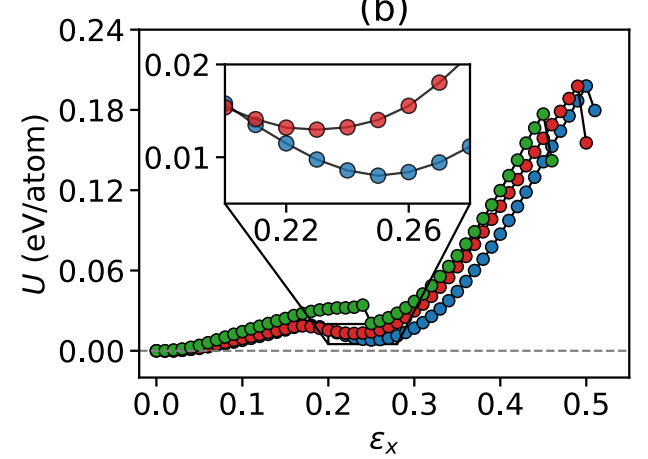

(d)

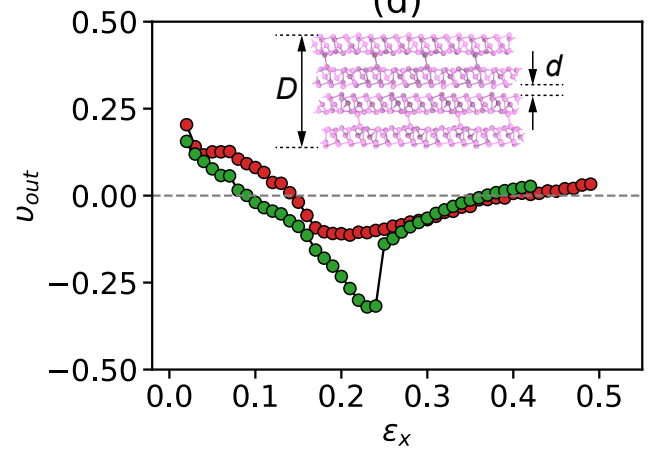

Figure 2: Evolution of (a) stress $\sigma_{x}$, (b) energy $U$, (c) in-plane Poisson's ratio $v_{i n}$, and (d) out-of-plane Poisson's ratio $v_{\text {out }}$ of monolayer, bilayer, and bulk VP during the tensile loading process. Here, the inset in (d) shows the thickness definition of bilayer VP.

For monolayer and bilayer VP structures, their abnormal negative-stiffness behavior at the second stage leads to twice appearances of zero stress under tension (see Fig. 2(a)). These two zero-stress points at the $\sigma_{x}-\varepsilon_{x}$ curves successively correspond to the maximum 
and the minimum points in the $U-\varepsilon_{x}$ curves shown in Fig. 2(b). Specifically, the minimum point of $U$ indicates the onset of phase transformation occurring in monolayer and bilayer $\mathrm{VP}$ structures due to tensile loading (see the inset in Fig. 2(b)), while the maximum point of $U$ corresponds to the energetic barrier of phase transformation. The new phases of monolayer and bilayer VP structures are metastable, because the energy of new phases is slightly higher than that of the parent phase. Different from monolayer and bilayer VP structures, a discontinuous variation of $U$ with growing $\varepsilon_{x}$ is observed in the bulk VP structure, since a sudden drop of $U$ occurs at $\varepsilon_{x} \approx 0.24$. This sudden drop of the energy of bulk VP is also accompanied by a sudden drop of its stress (see Fig. 2(a)). We attribute this energy and stress drop phenomenon to the interlayer slip occurring in the phase transformation process of bulk VP structure, which will be discussed in details in section 3.4.

Figure 2(c) and (d), respectively, shows the evolution of $v_{\text {in }}$ and $v_{\text {out }}$ with growing $\varepsilon_{x}$. It is noted that the calculation of $v_{\text {out }}$ relies on the material thickness, the definition of which is difficult for monolayer $2 \mathrm{D}$ materials. Therefore, $v_{\text {out }}$ of monolayer VP structure is not discussed here. It is found that $v_{i n}$ first increases with growing $\varepsilon_{x}$ when $\varepsilon_{x} \lesssim 0.2$. Afterwards, when $\varepsilon_{x} \gtrsim 0.2, v_{i n}$ begins to decrease as $\varepsilon_{x}$ increases. In other words, the change in $v_{\text {in }}$ with varied $\varepsilon_{x}$ generally exhibits a convex parabolic shape, which reaches the maximum value at $\varepsilon_{x} \approx 0.2$. Specifically, the maximum values of $v_{\text {in }}$ are $1.20,1.15$, and 1.08 for monolayer, bilayer, and bulk VP structures, respectively. All of these values are larger than one, which indicates that the magnitude of the responded transverse strain $\varepsilon_{y}$ is much larger than that of the applied longitudinal strain $\varepsilon_{x}$. As for bilayer and bulk VP structures, their $v_{\text {out }}$ has a positive value around 0.15 at the initial stage. However, when under tensile loading, $v_{\text {out }}$ can decrease from the initial positive value to a negative value with the transition occurring at the strains of 0.14 and 0.09 for bilayer and bulk VP structures, respectively. The maximum magnitudes of negative $v_{\text {out }}$ are -0.11 and -0.32 for bilayer and bulk VP structures, respectively, which are correspondingly observed at $\varepsilon_{x}=0.21$ and 0.23 . When compared with $v_{\text {out }}$ of other $2 \mathrm{D}$ materials with the out-of-plane auxetic property such as $v_{\text {out }}=-0.027$ for monolayer BP (Jiang and Park $(2014 \mathrm{~b})$ ), $v_{\text {out }}=-0.061$ for bilayer graphene (Li et al. (2020)), $v_{\text {out }}=-0.09$ for multilayer graphene (Woo et al. (2016)), $v_{\text {out }}=$ -0.09 for graphene/hexagonal $\mathrm{MoS}_{2}$ heterostructures (Li et al. (2020)) and $v_{\text {out }}$ in the range of [-0.111, -0.023] for graphene/hexagonal BN heterostructures (Li et al. (2021)), the present bulk VP structures are found to possess a more significant auxetic behavior under tensile loading especially when the tensile strain is in the range of 0.20 to 0.24 .

Based on $\sigma_{x}-\varepsilon_{x}$ and $U-\varepsilon_{x}$ curves shown in Figs. 2(a) and (b), the tension-induced phase transformation can be clearly detected in VP structures with different thicknesses. To determine the atomic structures of VP after the phase transformation, the new phases of monolayer, bilayer and bulk VP structures are obtained after performing the geometric optimization to their structures at the tensile strains of 0.24 for monolayer VP, 0.22 for bilayer $\mathrm{VP}$, and 0.25 for bulk VP, respectively, which correspond to critical strains at the onset of their phase transformation as shown in Fig. 2(b). Figure 3(a) shows atomic structures of the parent and transformed phases of monolayer VP. It can be seen that the transformed phase has an asymmetric structure, which shows a closed-pore structure enclosed by the cross sub- 

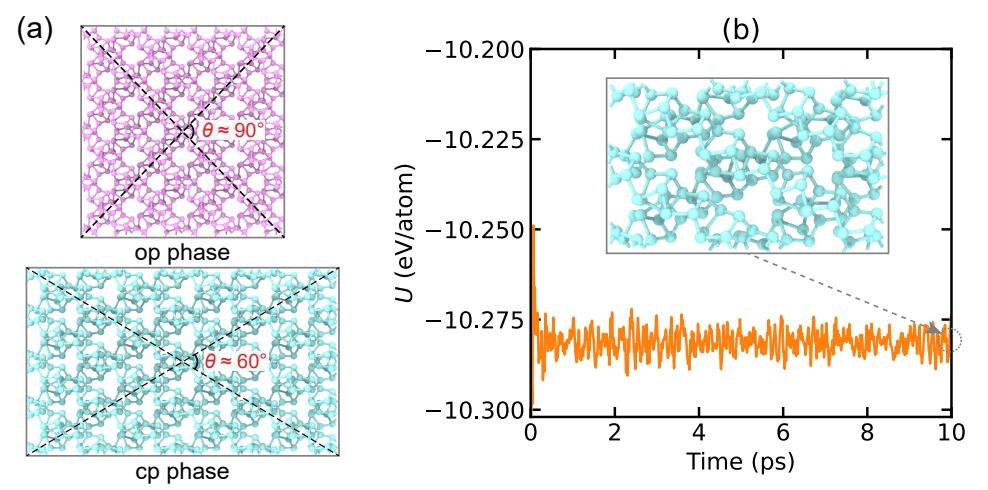

Figure 3: (a) The atomic structure of parent op phase and transformed cp phase of monolayer VP. Here, for clarity, the $4 \times 4 \times 1$ supercell is presented for both op and cp phases. (b) Total energy $U$ of the $2 \times 2 \times 1$ supercell of the cp phase of monolayer VP at $300 \mathrm{~K}$ calculated by AIMD simulations. The inset shows the structure of cp phase at 10 ps extracted from AIMD simulations.

nano rods. This is distinctly different with the open-pore structure of the parent phase of monolayer VP. The similar closed-pore structure is also found in the transformed phases of both bilayer and bulk VP. Thus, the parent phase and transformed phase of VP are named here as op (open-pore) phase and cp (closed-pore) phase, respectively. The lattice constants of op and cp phases are listed in Table 1. To quantitatively compare the structure changes induced by the phase transformation, we further calculate the in-plane angle $\theta$ between the cross sub-nano rods of op and cp phases. By assuming that the sub-nano rods before and after phase transformation remain straight, $\theta$ can be estimated as:

$$
\theta=\arctan \frac{2 L_{y}}{L_{x}}
$$

We find that $\theta$ of op and cp phases approximately equals to $90^{\circ}$ and $60^{\circ}$ (see Fig. 3(a)), respectively, indicating a structural transformation from the orthogonal network to a skew network for VP structures. In addition to the structure changes observed in the in-plane directions, the thickness (i.e., $L_{z}$ ) of bilayer and bulk VP structures is found to increase from $21.56 \AA$ and $20.56 \AA$ at the parent op phase to $22.09 \AA$ and $21.36 \AA$ at the transformed cp phase, respectively, which is consistent with the out-of-plane auxetic behavior mentioned before (see Fig. 2(d)).

To verify the thermodynamic stability of VP structures after phase transformation, taking the monolayer VP as an example, we preformed ab initio molecular dynamics (AIMD) simulations of its cp phase for 5000 steps. In doing this, a $2 \times 2 \times 1$ supercell based on the optimized structure containing 168 atoms was simulated within the NVT ensemble using Andersen thermostat at $300 \mathrm{~K}$, a time step of $2 \mathrm{fs}$, and a $1 \times 1 \times 1 k$-point mesh. The AIMD simulations were totally run for $10 \mathrm{ps}$. The energy evolution during the entire simulation is plotted in Fig. 3(b). It is found that the energy reaches equilibrium rapidly at the beginning of AIMD simulations and remains almost unchanged afterwards. Moreover, the structure obtained from AIMD simulations at the time of 10 ps (see insects in (Fig. 3(b))) is almost 
identical to the original cp phase of monolayer VP (Fig. 3(a)) without any bond breakages or disorders. These results indicate the thermodynamic stability of the transformed cp phase of VP structures.

Table 1: A comparison of lattice constants and mechanical properties of op and cp phases of monolayer, bilayer, and bulk VP structures. The lattice constants $l_{x}, l_{y}$, and $l_{z}$ are crystal lengths in the $x, y$ and $z$ directions, respectively, which all are in unit of $\AA$. The parameter $\alpha$ denotes the plane angle (from the top view) of the cross sub-nano rods as shown in Fig. 3(a), which is in unit of degree. The elastic constants $C_{11}$, $C_{22}, C_{12}$, and $C_{66}$ are obtained by Eq. 4. E, $G$, and $v_{i n}$ represent the Young's modulus, shear modulus, and in-plane Poisson's ratio, respectively. All the elastic constants and moduli are in unit of GPa.

\begin{tabular}{ccccccc}
\hline \hline \multirow{2}{*}{$\mathrm{VP}$} & \multicolumn{2}{c}{ Monolayer } & \multicolumn{2}{c}{ Bilayer } & \multicolumn{2}{c}{ Bulk } \\
\cline { 2 - 7 } & op phase & cp phase & op phase & cp phase & op phase & cp phase \\
\hline$l_{x}$ & 8.89 & 11.10 & 9.03 & 11.07 & 9.10 & 11.23 \\
$l_{y}$ & 9.00 & 6.60 & 8.89 & 6.72 & 8.92 & 6.73 \\
$l_{z}$ & $/$ & $/$ & 21.56 & 22.09 & 20.56 & 21.36 \\
$\alpha$ & 89.2 & 61.4 & 89.2 & 62.6 & 88.8 & 61.8 \\
\hline$C_{11}$ & 68.24 & 146.35 & 69.69 & 132.77 & 78.86 & 141.12 \\
$C_{22}$ & 68.24 & 68.33 & 69.69 & 57.85 & 73.08 & 74.81 \\
$C_{12}$ & 55.41 & 45.56 & 53.61 & 44.72 & 53.59 & 40.02 \\
$C_{66}$ & 57.84 & 51.02 & 57.00 & 50.07 & 55.43 & 46.84 \\
$E$ & 23.25 & 115.98 & 28.45 & 98.20 & 39.57 & 119.71 \\
$G$ & 57.84 & 51.02 & 57.01 & 50.07 & 55.43 & 46.84 \\
$v_{i n}$ & 0.81 & 0.67 & 0.77 & 0.77 & 0.73 & 0.53 \\
\hline \hline
\end{tabular}

\subsection{Anisotropic elastic properties}

In section 3.1, we revealed the tension-induced op-to-cp phase transformation in VP structures and further demonstrated the thermodynamic stability of the transformed cp phase at the finite temperature. In this section, we further investigate the elastic properties of $\mathrm{cp}$ phases and compare the mechanical properties of the transformed phase to those of its parent counterpart.

Since monolayer and multi-layer VP structures in current work can be considered as 2D rectangular system, we only consider the 2D elastic constants of VP under the in-plane stress condition using Viogt scheme (Voigt (1928)). Under this circumstance, the constitutive equation of VP can be written as:

$$
\left[\begin{array}{l}
\sigma_{1} \\
\sigma_{2} \\
\sigma_{6}
\end{array}\right]=\left[\begin{array}{ccc}
C_{11} & C_{12} & 0 \\
C_{12} & C_{22} & 0 \\
0 & 0 & C_{66}
\end{array}\right]\left[\begin{array}{l}
\varepsilon_{1} \\
\varepsilon_{2} \\
\varepsilon_{6}
\end{array}\right],
$$

where $\sigma_{i}$ and $\varepsilon_{j}$ indicate the stress and strain, $C_{i j}$ is the elastic constant tensor, $i, j=1,2$, and 6 in the Voigt notation correspond to $x x, y y$, and $x y$ in tensor notation, respectively. Based on the elastic constants, one can rewrite the elastic constants in a new basis set and 
further calculate the orientation-dependent Young's modulus $E(\theta)$, shear modulus $G(\theta)$, and Poisson's ratio $v_{i n}(\theta)$ of $2 \mathrm{D}$ rectangular system based on the following formula (Jasiukiewicz et al. $(2008,2010))$ :

$$
\begin{aligned}
& E(\theta)=1 /\left(S_{11} a^{4}+S_{22} b^{4}+\left(S_{66}+2 S_{12}\right) a^{2} b^{2}\right), \\
& \left.G(\theta)=1 /\left(4\left(S_{11}+S_{22}-2 S_{12}\right) a^{2} b^{2}+S_{66}\left(a^{2}-b^{2}\right)^{2}\right)\right), \\
& v_{i n}(\theta)=-E(\theta)\left(\left(S_{11}+S_{22}-S_{66}\right) a^{2} b^{2}+S_{12}\left(a^{4}+b^{4}\right)\right),
\end{aligned}
$$

with

$$
a=\cos (\theta), b=\sin (\theta),
$$

in which $\theta$ denotes the angle between the new axis and the original $+x$ axis. $S_{11}, S_{22}$, and $S_{66}$ in Eq. 5 are compliance constants that can be obtained by inverting the elastic constants matrix as defined in Eq. 4.

We calculated the elastic constants of VP structures on the basis of stress-strain relationship (Eq. 4) with the aid of VASPKIT package (Wang et al. (2021)). Each elastic constant component was determined by using the first-order derivative of the stress-strain curve that contains nine small strains ranging from $-2 \%$ to $2 \%$ in the elastic regime. All elastic constant components together with $E, G$, and $v_{i n}$ in the original $+x$ axis are listed in Table 1 , while the orientation-dependent $E(\theta), G(\theta)$, and $v_{i n}(\theta)$ obtained by Eq. 5 are presented in Fig. 4 .

The elastic constants obtained above can be employed to examine the mechanical stability of 2D VP structures including both op and cp phases. On the basis of the Born stability criteria (Born (1940)), Maździarz (2019) proposed the stability criteria for 2D rectangular lattices with the following forms:

$$
\begin{aligned}
& \frac{1}{2}\left(C_{11}+C_{22}+\sqrt{4 C_{12}^{2}-\left(C_{11}-C_{22}\right)^{2}}\right)>0, \\
& \frac{1}{2}\left(C_{11}+C_{22}-\sqrt{4 C_{12}^{2}-\left(C_{11}-C_{22}\right)^{2}}\right)>0, \\
& C_{66}>0 .
\end{aligned}
$$

Substituting the elastic constants listed in Table 1 to Eq. 7, we find that the stability criteria is satisfied for all VP structures, demonstrating the mechanical stability of the parent op phase and transformed cp phase for monolayer, bilayer, and bulk VP.

From the elastic constants listed in Table 1, one can clearly find that the parent op phases of all VP structures show the similar mechanical properties in both $x$ and $y$ directions. However, a strongly different mechanical behavior is observed in the cp phases of VP, whose $C_{11}$ is much larger than $C_{22}$. This different mechanical properties observed between op and cp phases of VP structures can be easily understood by their structures before and after phase transformation as shown in Fig. 3(a). In the op phase, VP has a symmetry structure along $x$ and $y$ directions, while this symmetry is broken in the cp phase. In addition, regarding each component of elastic constants of VP before and after phase transformation, the most significant difference is observed in $C_{11}$ of op and cp phases. Taking the monolayer 
VP for example, $C_{11}$ of its cp phase is $146.35 \mathrm{GPa}$, which is more than twice the value of its op counterpart. The enhancement in $C_{11}$ induced by phase transformation further leads to a much higher $E$ in cp phase than that in op phase. As listed in Table 1, the Young's moduli of monolayer, bilayer and bulk VP grow from 23.25, 28.45 and 39.57 GPa to 115.98, 98.20 and 119.71 GPa, respectively, after the op-to-cp phase transformation. This phase transformation-induced strengthening effect also can be observed in the $\sigma_{x}-\varepsilon_{x}$ curves shown Fig. 2(a), in which the slope of $\sigma_{x}-\varepsilon_{x}$ curve of cp phase at the initial tension stage is much larger than that of the op phase similarly at initial tension stage. In addition, the shear modulus of all VP structures is reduced after the phase transformation, while in this process a decrease of the in-plane Poisson's ratio is observed in monolayer and bulk VP.

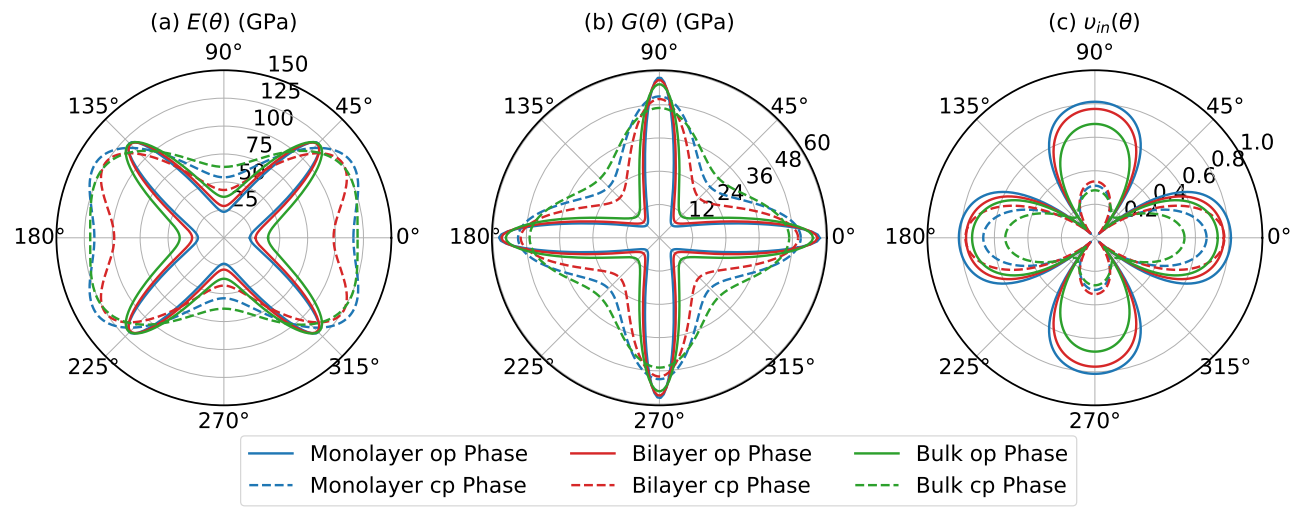

Figure 4: Orientation-dependent (a) Young's modulus $E(\theta)$, (b) shear modulus $G(\theta)$, and (c) in-plane Poisson's ratio $v_{i n}(\theta)$ of monolayer, bilayer, and bulk VP structures obtained by Eq. 5 . Here, the solid line and dash line denote the results of op and cp phases for each VP structure, respectively.

Table 2: The maximum value, minimum value, and anisotropy ratio of $E(\theta), G(\theta)$, and $v_{i n}(\theta)$, which are extracted from Fig. 4 for op and cp phases of monolayer, bilayer, and bulk VP. The subscripts "max" and "min" denote the corresponding maximum value and minimum value, respectively. $A_{E}, A_{G}$, and $A_{v}$ denote the anisotropy ratio of $E(\theta), G(\theta)$ and $v_{i n}(\theta)$, respectively. All elastic moduli are in the unit of GPa, while the others are dimensionless.

\begin{tabular}{ccccccc}
\hline \hline \multirow{2}{*}{$\mathrm{VP}$} & \multicolumn{2}{c}{ Monolayer } & \multicolumn{2}{c}{ Bilayer } & \multicolumn{2}{c}{ Bulk } \\
\cline { 2 - 7 } & op phase & cp phase & op phase & cp phase & op phase & cp phase \\
\hline$E_{\max }$ & 119.59 & 132.93 & 118.47 & 125.56 & 119.48 & 125.64 \\
$E_{\min }$ & 23.25 & 54.15 & 28.45 & 42.79 & 36.67 & 63.67 \\
$A_{E}$ & 5.14 & 2.45 & 4.16 & 2.93 & 3.26 & 1.98 \\
\hline$G_{\max }$ & 57.87 & 51.02 & 57.00 & 50.07 & 55.43 & 46.84 \\
$G_{\min }$ & 6.42 & 25.92 & 8.04 & 20.28 & 11.16 & 30.26 \\
$A_{G}$ & 9.02 & 1.97 & 7.09 & 2.47 & 4.97 & 1.55 \\
\hline$v_{\max }$ & 0.81 & 0.67 & 0.77 & 0.77 & 0.73 & 0.53 \\
$v_{\min }$ & 0.03 & 0.11 & 0.04 & 0.06 & 0.08 & 0.16 \\
$A_{v}$ & 24.56 & 5.96 & 19.61 & 13.63 & 9.60 & 3.24 \\
\hline \hline
\end{tabular}


From Fig. 4, it can be found that, consistent with their structures, the mechanical properties including $E(\theta), G(\theta)$ ), and $v_{i n}(\theta)$ of op and cp phases exhibit 4-fold and 2-fold rotation symmetries, respectively. Therefore, we only need to discuss the distribution of elastic properties within $0^{\circ} \leq \theta \leq 45^{\circ}$ for op phase and those within $0^{\circ} \leq \theta \leq 90^{\circ}$ for $\mathrm{cp}$ phase. The maximum value, minimum value, and corresponding anisotropy ratio of $E(\theta)$, $G(\theta)$, and $v_{i n}(\theta)$ extracted from Fig. 4 are listed in Table 2. Here, the anisotropy ratio is defined as the ratio of the maximum value to the minimum value. From Table 2, we can see that the changes in all mechanical properties after the op-to-cp phase transformation are qualitatively similar for monolayer, bilayer, and bulk VP, though a slight quantitative difference is observed among different VP materials.

Regarding $E(\theta)$ of op phase, its maximum value $E_{\max }$ is found to locate at $45^{\circ}$ that corresponds to the direction along sub-nano rods (see Fig. 3(a)), while its minimum value $E_{\text {min }}$ is found at $0^{\circ}$ that is the $x$ axis. Specifically, $E_{\max }$ and $E_{\text {min }}$ of op phase of monolayer VP are, respectively, $119.59 \mathrm{GPa}$ and $23.25 \mathrm{GPa}$, resulting in a significant anisotropy ratio of $A_{E}=5.14$. This significant anisotropy effect originates from the different deformation mechanisms of op phase of VP along these two directions. The deformation in the $45^{\circ}$ direction is majorly attributed to the extension of bonds in the component sub-nano rods, i.e., -[P2]-[P8]-[P2]-[P9]- repeating units as shown in Fig. 1, which makes VP have the largest stiffness in this direction. The deformation along the $0^{\circ}$ direction is mainly induced by the change of in-plane angle $\alpha$ as defined in Fig. 3(a), i.e., the relative rotation between cross rods. As a result, in this direction VP will have a relatively small stiffness. The deformation mechanisms of VP will be further investigated in details in the section 3.3. Similar to its op counterpart, $E_{\max }$ of the cp phase of $\mathrm{VP}$ is found at the directions along the sub-nano rods, i.e., now at $\theta \sim 30^{\circ}$ as shown in Fig. 3(a). However, $E_{\text {min }}$ of $\mathrm{cp}$ phase is found at the $y$ direction of $90^{\circ}$, which is totally different to the $x$ direction of its op counterpart. It also can be found that, $A_{E}$ of the cp phase ranges from 1.98 to 2.93, which is much smaller than that of op phase having the range from 3.26 to 5.14. This result demonstrates a distinct distribution of $E(\theta)$ between these two phases of VP.

The op and cp phases of VP have the similar distribution of $G(\theta)$, which has the maximum at the $x$ or $y$ direction and the minimum at the direction of $45^{\circ}$. But the anistropy of $G(\theta)$ is different for op and cp phases. Specifically, $A_{G}$ of op phase is in the range of $4.97 \sim 9.02$, which is much higher than the value of its cp counterpart that ranges from 1.55 to 2.47. The significantly lower $A_{G}$ of cp phase is mainly attributed to the much larger $G_{\min }$ after the op-to-cp phase transformation. For instance, $G_{\min }$ of monolayer VP within the cp phase is 25.92 GPa, which is four times larger than 6.42 GPa of its counterpart within the op phase.

We find in Fig. 4 that $v_{i n}$ is very close to zero at $45^{\circ}$ for both op and cp phases of VP, which is especially apparent for the op phase of monolayer and bilayer VP with $v_{\text {min }}$ being only 0.03 and 0.04 , respectively. This near-zero Poissons ratio found in the specific in-plane direction of VP structures indicates that when VP is stretched or compressed in this direction, the length of its transverse direction can keep almost unchanged. This zero in-plane Poissons ratio together with the aforementioned negative out-of-plane Poissons 
ratio (see Fig. 2(d)) indicates that the VP structures are excellent auxetic 2D materials, which are thus expected to have widespread applications in fields such as medicine/tissue engineering and aerospace (Rothenburg et al. (1991); Evans and Alderson (2000)). The maximum Poisson's ratio $v_{\max }$ of op and cp phases is found at the $0^{\circ}(x$ axis) direction. Specifically, values of $v_{\max }$ are $0.81,0.77$, and 0.73 for the op phase of monolayer, bilayer and bulk VP structures, respectively, while the corresponding values of their cp counterparts are $0.67,0.77$, and 0.53 . These values are consistent with the results obtained from the tensile simulation as shown in Fig. 2(c). Moreover, the in-plane Poisson's ratio of op phase of VP structures is found to generally decrease with increasing thickness. This thickness dependence of Passion's ratio is attributed to the following mechanism. In bulk VP, the interaction between layers can constrain the out-of-plane deformation and further reduce the in-plane contraction along the $y$ direction when it is stretched along the $x$ direction, which finally leads to a relatively small $v_{i n}$. Due to the absence of neighboring layers, monolayer VP has more freedoms to deform in the out-of-plane direction, which results in a relatively large $v_{i n}$. The degree of out-of-plane constrains in bilayer VP structures is between that of their monolayer and bulk counterparts, leading to a $v_{\text {in }}$ between the values of monolayer and bulk VP as shown in Fig. 2(c). The similar mechanism was also demonstrated by Pang and $\mathrm{Li}$ (2021) in the $\mathrm{Bi}_{2} \mathrm{O}_{2}$ Se system.

\subsection{Extremely large in-plane Poisson's ratio}

To shed light on the structural evolution of VP structures under tension and the accompanied phase transformation as discussed above, in this section the in-plane deformation mechanism of VP structures is investigated based on the geometry analysis together with the mechanical modeling. Efforts are made to reveal the origin of extremely large in-plane Poisson's ratio found in VP structures under uniaxial tension.

Considering the unique cross structure of VP whose component sub-nano rods are linked by [P9] units (see Fig. 1), a VP cell can be approximately treated as a mechanical framework. As shown in Fig. 5(a), in the framework model the sub-nano rods are treated as straight rods, while the [P9] unit is treated as the hinge linking two cross rods. Based on this mechanical framework model, the overall deformation of VP structures under tension is attributed to two deformation mechanisms that are, respectively, elongation and rotation of rods. Assuming the elongation and rotation angle of rods are $d s$ and $d \theta$, respectively, we can obtain the following equations from the general Hooke's law

$$
\begin{aligned}
& \frac{F s}{2} \cos (\theta / 2)=K_{s} d s, \\
& \frac{F s}{2} \sin (\theta / 2)=K_{r} d \theta,
\end{aligned}
$$

where $F$ is the total tensile force $F$ acting on the framework, $\frac{F s}{2} \cos (\theta / 2)$ and $\frac{F s}{2} \sin (\theta / 2)$ are, respectively, the stretching force and rotation moment acting on the rod components, and $K_{s}$ and $K_{r}$ are the generalized force constants. 
(a)

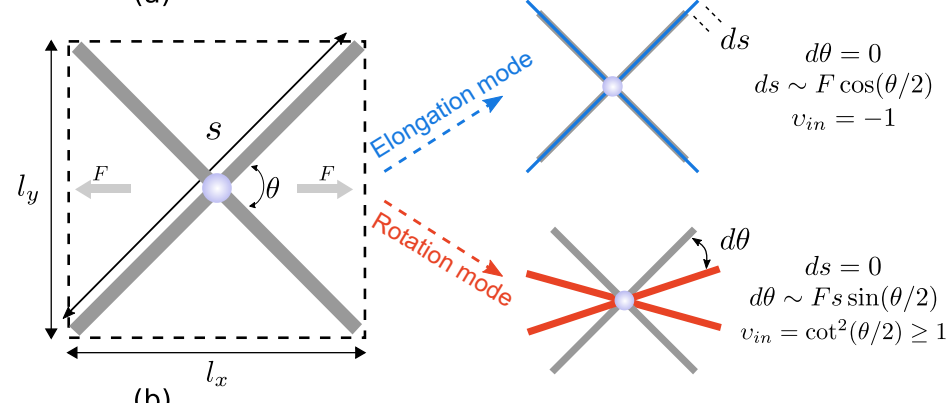

(b)

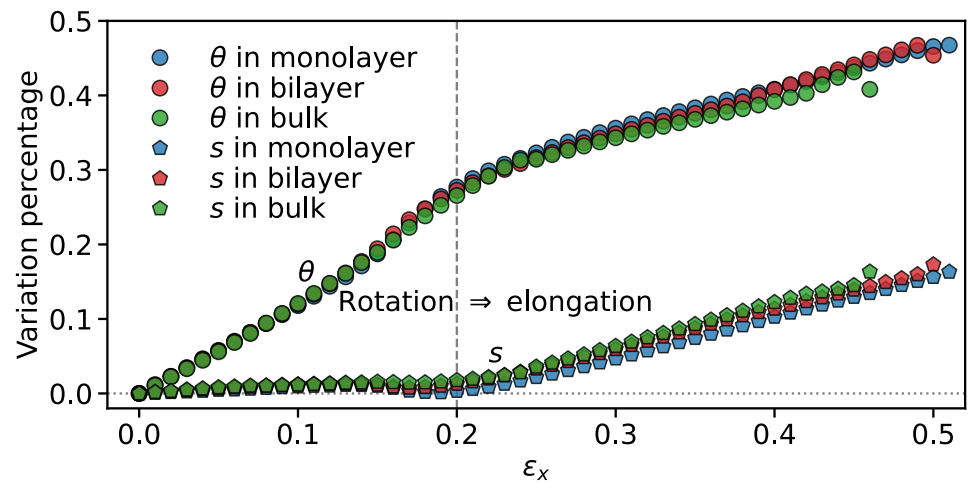

Figure 5: (a) Illustration of the deformation mechanism of VP structures under tensile loading. Here, the tensile strain is attributed to two deformation modes including rotation and elongation. Here, $F$ represents the tensile force acting on the framework, $s$ represents the length of sub-nano rods along diagonal direction and $\theta$ is the in-plane angle between two cross diagonal rods. (b) The variation percentage of $s$ and $\theta$ of mononlayer, bilayer, and bulk VP structures with respect to the tensile strain. The variation percentage of $S$ and $\theta$ are, respectively, calculated as $\Delta s / s$ and $\Delta \theta / \theta$.

Meanwhile, the lengths of VP cell $l_{x}$ and $l_{y}$ in the $x$ and $y$ directions, respectively, are

$$
\begin{aligned}
& l_{x}=s \cos (\theta / 2), \\
& l_{y}=s \sin (\theta / 2) .
\end{aligned}
$$

From Eq. 9 we can further have

$$
\begin{aligned}
& d l_{x}=\cos (\theta / 2) d s-\frac{s}{2} \sin (\theta / 2) d \theta, \\
& d l_{y}=d s \sin (\theta / 2)+\frac{s}{2} \cos (\theta / 2) d \theta .
\end{aligned}
$$

According to Eq. 2, the in-plane Poisson's ratio $v_{i n}$ contributed by the elongation and rotation of component rods can be written as

$$
v_{i n}=-\frac{d l_{y} / l_{y}}{d l_{x} / l_{x}}=-\frac{d s / s+\frac{1}{2} \cot (\theta / 2) d \theta}{d s / s-\frac{1}{2} \tan (\theta / 2) d \theta}
$$

Specifically, when only considering the contribution of the elongation of rod components under the assumption of $d \theta=0, v_{i n}=-1$. In turn, when the contribution of elongation 
is excluded under the assumption of $d s=0$, the in-plane Poisson's ratio only due to the rotation of component rods is $v_{i n}=-\cot ^{2}(\theta / 2)$.

Figure 5(b) illustrates the changes in length and rotation angle of the component subnano rods during the tension process of VP structures, which can be used to measure the contribution of elongation and rotation to the overall deformation of VP structures in this process. Here, the value of $\theta$ is obtained by Eq. 3, while the value of $s$ can be directly estimated as $s=\sqrt{l_{x}^{2}+l_{y}^{2}}$. As for all VP structures considered here, the changes in their $\theta$ and $s$ are identical to each other. Specifically, under the initial deformation with a strain smaller than $0.2, \theta$ increases linearly with growing strain, while $s$ keeps almost unchanged in this process. However, when the strain grows greater than 0.2 , a more significant increase is abruptly observed in $s$ accompanied with a slower increase of $\theta$, which makes the change in $s$ become comparable to that in $\theta$. This result reveals that at a strain smaller than 0.2 , the deformation of VP structures is only dominated by the rotation of their component sub-nano rods. However, at a strain larger than 0.2 , the elongation and rotation of sub-nano rods begin to contribute equally to the overall deformation of VP structures.

The change in the deformation mechanism observed at the strain of 0.2 can be explained as follows. In the entire tension process, $\theta$ decreases monotonically as the tensile strain increases. Meanwhile, the monotonic decrease and increase are, respectively, observed in the rotation moment $F s \sin (\theta / 2)$ and the stretching force $F s \cos (\theta / 2)$ as shown Eq.8. Thus, at the beginning stage of the tension process with the largest $\theta$ around $45^{\circ}$ as shown in Fig. 3(a), the rotation moment approximately equals to the stretching force. However, the deformation of VP structures under tension prefers the rotation rather than elongation of their component component sub-nano rods, since the deformation is majorly governed by the deformation mode with a lower pathway energy (Jiang et al. (2016). As $\theta$ decreases with growing strain, the stretching force and the rotation moment will, respectively, increase and decrease during the tension process. Specifically, at the strain of 0.2 , the impact of the increased stretching force will become comparable to that of the decreased rotation moment. This competition between two deformation modes finally results in the change in the deformation mechanism of VP structures observed in Fig.5(b).

The deformation mechanism demonstrated here can be further adopted to explain the evolution of $v_{\text {in }}$ of VP structures under tension and the origin of extremely large $v_{\text {in }}$ observed at $\varepsilon_{x} \approx 0.2$. When $\varepsilon_{x} \lesssim 0.2$, the deformation is fully dominated by rotation of the subnano rods. Thus, $v_{i n}=-\cot ^{2}(\theta / 2)$ according to our model. Under this circumstance, $v_{i n}$ increases from $\sim 1.0$ to $\sim 2.46$ as $\theta$ decreases from $\sim 90^{\circ}$ to $\sim 65^{\circ}$. This prediction is qualitatively similar to $v_{i n}$ shown in Fig. 2(c) with different $\theta$. However, $v_{\text {in }}$ obtained from DFT calculations that is in the range of $\sim 0.75$ to $\sim 1.20$ is much lower the theoretically predicted value. This quantitative difference can be understood by the fact that sub-nano rods assumed as rigid and straight rods in our mechanical model ignore the contributions of other distortions such as twist and bending in the realistic tension process; however, the twist and bending distortions can accommodate parts of the lateral deformation under uniaxial tension, which thus can dramatically reduce the magnitude of $v_{\text {in }}$ predicted by the 
mechanical model. When $\varepsilon_{x} \gtrsim 0.2$, in addition to the rotation, the elongation of sub-nano rods also becomes another important factor responsible for the deformation of VP structures. However, according to our model, the elongation of sub-nano rods corresponds to a constant negative $v_{i n}$ of -1 , leading to a synchronous reduction of $v_{i n}$ as shown in see Fig. 2(c).

In addition to $v_{i n}$, the deformation mechanism illustrated above can be also used to reveal the evolution of three stages in $\sigma_{x}-\varepsilon_{x}$ curves shown in Fig. 2(a). At $\varepsilon_{x} \lesssim 0.2$ where the rotation of sub-nano rods dominates the overall deformation, the stress changes gently with varied strain. But at $\varepsilon_{x} \gtrsim 0.2$ where the elongation of sub-nano rods starts to take part in the overall deformation, the stress becomes to sharply increase with growing strain. We attribute this difference to the fact that the bond stretching of sub-nano rods is much harder than the rotation between sub-nano rods, corresponding to a much stiffer force constant $K_{s}$ compared to $K_{r}$. This also explains why Young's modulus of cp phase structures is much larger than that of their op phase counterparts (see Table 1). It is noticed that a slight decrease of the variation percentage of $s$ is observed at the strain between 0.15 and 0.20 in Fig.5(b), which indicates the instability of the transition between aforementioned two deformation modes. This observation is consistent with the negative-stiffness stage observed in $\sigma_{x}-\varepsilon_{x}$ curves, which could be the factor responsible for op-to-cp phase transformation.

The Young's modulus $E$ of parent phase of VP structures calculated in current work is less than $40 \mathrm{GPa}$ (see Table 1), indicating an extremely flexible behavior of VP structures under uniaxial tension. Specifically, $E$ of monolayer VP is estimated as $23.25 \mathrm{GPa}$, which is an order of magnitude lower than other monolayer 2D materials such as $1 \mathrm{TPa}$ of graphene with an equivalent thickness of 3.40 $\AA$ (Lee et al. (2008)), 295.57 GPa of $\mathrm{MoS}_{2}$ with an equivalent thickness of $6.09 \AA$ (Bertolazzi et al. (2011)), and 407.62 GPa of black phosphorene with an equivalent thickness of $5.25 \AA$ (Zhang et al. (2022)). As we explained above, this extremely flexible behavior of VP originates from the rotation between cross sub-nano rods without bond stretching at the initial stage of uniaxial tension process. It is noticed that, in a very recent experiment (Zhang et al. (2021)), $E$ of monolayer VP measured by AFMbased nanoindentation is $1.47 \mathrm{TPa}$, which is much larger than the value (1 TPa) of graphene that is recognized as the strongest material. Herein, we attribute this significant difference between $E$ obtained from the present calculations and previous experiments to the strongly anisotropic mechanical response of VP structures under different loading conditions. Due to its complex cross structures demonstrated previously by Zhang et al. (2021), VP shows an extremely large deformation resistance with respect to the (out-of-plane) normal loading, which may lead to the very high $E$ measured in the nanoindentation experiments (Zhang et al. (2021)). However, when under the (in-plane) uniaxial loading, the overall deformation is caused by the rotation of sub-nano rods as demonstrated above, which results in an ultrahigh flexibility property and correspondingly a very low E. Therefore, the value of 23.25 GPa calculated here and the value of $1.47 \mathrm{TPa}$ obtained from the previous nanoindentation experiments actually correspond to Youngs modulus of monolayer VP under in-plane tension and out-of-plane bending, respectively. The significantly different abilities of VP to resist stretching and bending deformations make VP be a stretchable but impact-resistant material, which will open the doors to the design of novel structural and functional materials 
in the future.

\subsection{Negative out-of-plane Poisson's ratio}

We have demonstrated that both bilayer and bulk VP structures have auxetic properties, i.e., negative $v_{\text {out }}$, when the strain is larger than a critical value (see Fig.2(d)). The maximum magnitude of $v_{\text {out }}$ ultimately can reach -0.32 for bulk VP, while the maximum value is -0.11 for bilayer VP. In this section, the underlying mechanism of auxetic properties found in VP structures is revealed based on geometry analysis together with the orbital theory.

(b) Contributed by intra-layer rotation mode

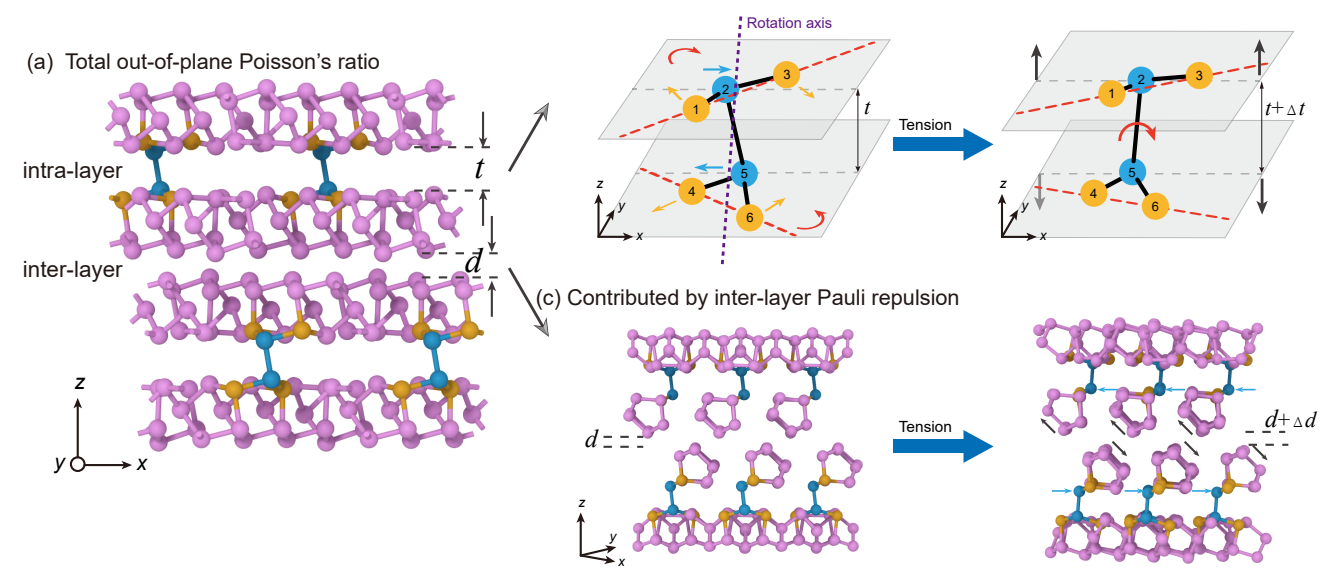

Figure 6: (a) The illustration of the out-of-plane Poisson's ratio $v_{\text {out }}$ of bilayer and bulk VP structures contributed by the variation of intra-layer spacing $t$ and inter-layer spacing $d$. Here, the atoms forming linked bonds through intra-layer spacing are highlighted in blue, while the atoms connecting the blue bonds and sub-nano rods are highlighted in yellow. (b, c) The illustration of intra-layer rotation and inter-layer Pauli repulsion deformation.

As shown in Fig. 6(a), the overall out-of-plane deformation contains two parts including the variation of intra-layer spacing $t$ and that of inter-layer spacing $d$. Specifically, it is emphasized that the atoms in individual [P9] units (as highlighted in Fig. 6) can form the covalent bonds, which result in the intra-layer spacing. These atoms can move like a hinge facilitating the rotation of two connected sub-nano rods, which thus plays a very important role in the auxetic phenomenon of bilayer and bulk VP structures.

To show the underlying mechanism of the variation of intra-layer spacing $t$, in Fig. 6(b) we specifically illustrate the hinge structure composed of six atoms, in which the sub-nano rods are represented by red dash lines. We find that the linked bond composed of atoms 2 and 5 (in blue) deviates from the central axis of rotation, which is represented as the purple dash line connecting midpoints of 1-3 bond and 4-6 bond (in yellow). Under this circumstance, the tension along the $x$ axis would lead to the in-plane rotation. Specifically, atoms 1 and 3 would rotate clockwise around the central axis, while atoms 4 and 6 would rotate counterclockwise around the central axis. The in-plane rotation of these atoms is also accompanied with the movement of atoms 2 and 5 along two opposite directions, which 
further leads to the rotation of 2-5 bond vertically, i.e., parallel to the central axis. This rotation finally results in the increment of intra-layer spacing $t$ (see right part of $6(\mathrm{~b})$ ) and thus a negative $v_{\text {out }}$.

Besides the variation of intra-layer spacing $t$, the negative $v_{\text {out }}$ can be also induced by the inter-layer Pauli repulsion, i.e., the variation of inter-layer spacing $d$ as shown in Fig. $6(\mathrm{c})$. It is found that the rotations of $2-5$ bonds locating at the upper and lower layer are in opposite directions as shown by the blue arrows, which further leads to the slip of sub-nano rods near the spacing $d$ (black arrows). Since the $\mathrm{P}$ atom in all VP structures has a fully filled $p_{z}$ orbital, the slip phenomenon shown in Fig. 6(c) is accompanied with the large overlap of $p_{z}$ orbitals, which finally results in the increment of spacing $d$ and thus a negative $v_{\text {out }}$.
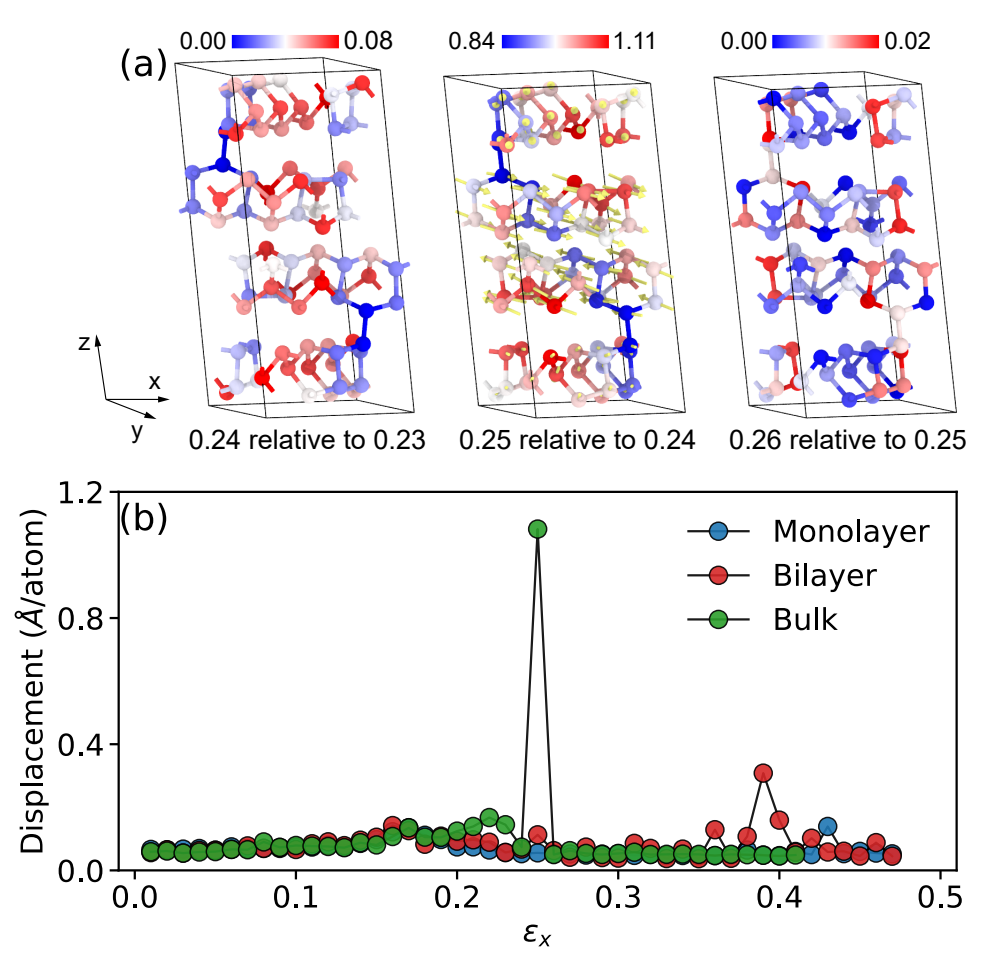

Figure 7: (a) Relative atomic displacement of bulk VP structure at strains of 0.24 (left), 0.25 (middle), and 0.26 (right). The atoms are colored from blue to red according to their corresponding displacement magnitudes. The yellow arrows show the atomic displacement vectors. The atomic displacement is in the unit of $\AA$. Here, the displacement vectors are not shown in the structures at strains of 0.24 and 0.26 because their magnitudes are extremely small. (b) The average value of relative atomic displacement of monolayer, bilayer, and bulk VP as a function of uniaxial tensile strain $\varepsilon_{x}$.

Although both bilayer and bulk VP structures exhibit the negative $v_{\text {out }}$ under tension, as shown in Fig. 2(d), the variation of $v_{\text {out }}$ of bulk VP during the tension process is discontinuous at $\varepsilon_{x} \approx 0.24$ accompanied by a step rise, which is distinct from the continuous change of $v_{\text {out }}$ of bilayer VP. To understand the origin of the step rise observed in the $v_{\text {out }}$ of bulk VP, in Fig.7(a) we show the relative atomic displacements of bulk VP structures at different 
(a) Monolayer VP

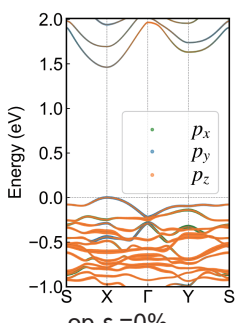

op $\varepsilon_{x}=0 \%$

(b) Bilayer VP

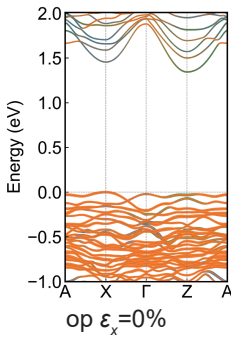

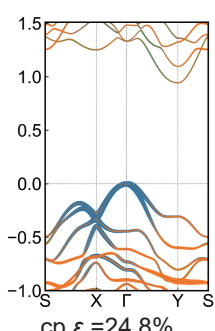

cp $\varepsilon_{x}=24.8 \%$

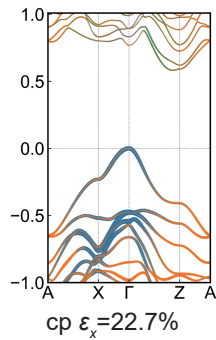

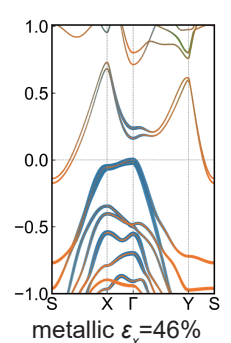

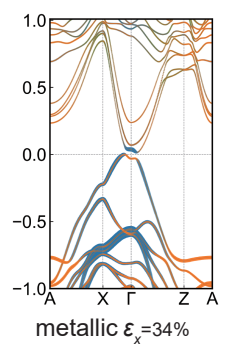

(d) Band gap evolution under tension

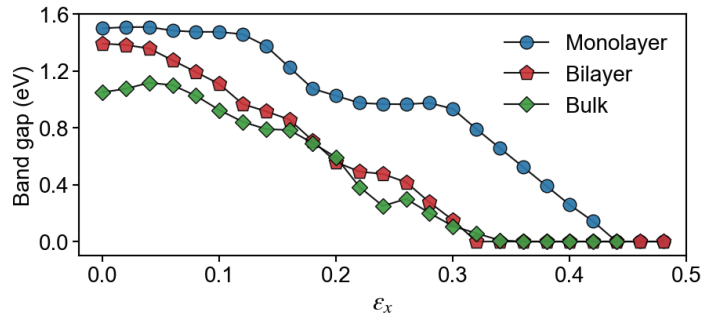

(c) Bulk VP
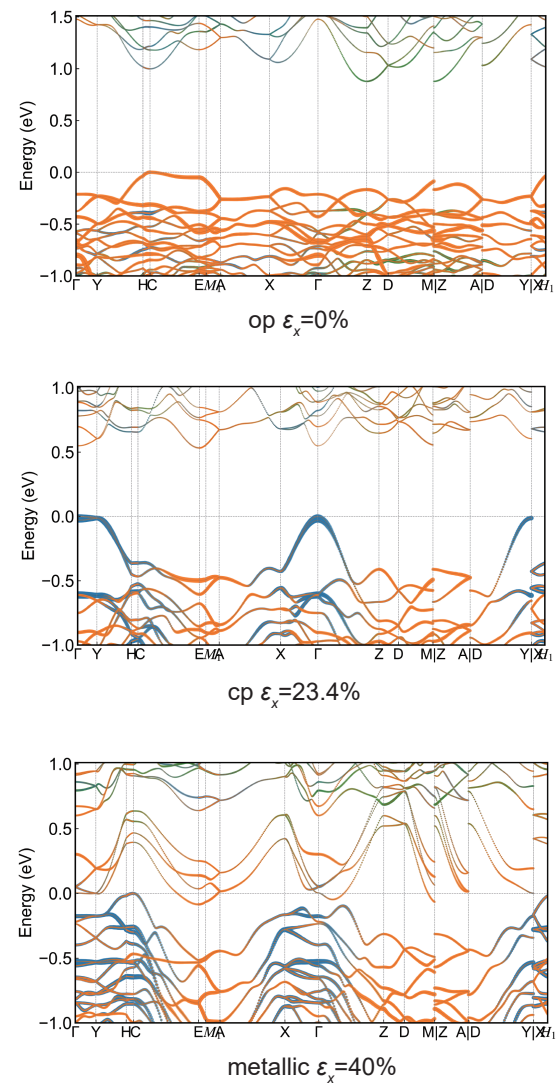

Figure 8: Band structures of (a) monolayer, (b) bilayer and (c) bulk VP under zero strain (op phase), strain of the maximum negative $v_{\text {out }}$ (cp phase), and strain of semiconductor-to-metal transition (metallic phase), respectively. The green, blue, and orange lines represent the contributions of the $p_{x}, p_{y}$, and $p_{z}$ orbitals, respectively. (d) Band gap of monolayer, bilayer, and bulk VP as a function of tensile strain $\varepsilon_{x}$, which indicates the semiconductor-to-metal transition.

strains. From Fig.7(a) it can be seen that the relative change between atomic displacements at $\varepsilon_{x}$ of 0.24 and 0.23 is trivial. The similar trivial atomic displacement change is also found between the structures at $\varepsilon_{x}$ of 0.26 and 0.25 . However, an interlayer slip process accompanied with the significant relative atomic displacements is found between the structures at $\varepsilon_{x}$ of 0.25 and 0.24 . In Fig.7(b), we further compare the average value of relative atomic displacement of monolayer, bilayer, and bulk VP during the whole tension process. It is found that the variation of atomic displacement of bilayer and monolayer VP in this process is generally smooth, while one peak locating at $\varepsilon_{x}$ of 0.24 is observed in bulk VP, which corresponds to the interlayer slip as shown in Fig.7(a). As for bulk VP, the interaction between layers restricts the smooth motion of interlayer atoms existing in monolayer and bilayer VP structures (see Fig.7(b)). The resulting energy barrier in bulk VP finally leads to a maximum value of negative $v_{\text {out }}=-0.32$ before the abrupt slip process.

In order to further understand the physical source of the negative Poisson's ratio phe- 
nomenon, we calculated the energy band structures of monolayer, bilayer and bulk VP. When the strain is absent, the contribution of the $p_{z}$ orbital of $\mathrm{P}$ atom dominates near the Fermi level (see op phase in Fig. 8(a-c)), and there is no strong interaction between the $p_{z}$ orbital and other orbitals. With the increase of tensile strain, the contribution of the $p_{y}$ orbital increases rapidly near the Fermi level (see cp phase in Fig. 8(a-c)). $p_{z}$ and $p_{y}$ orbitals show a strong hybridization. Simultaneously, VP shows a significant negative Poisson's ratio phenomenon. This further reveals the microscopic origin of the negative Poisson's ratio in VP structures from the perspective of quantum theory, as illustrated in Fig. 6(b). Moreover, by using the tight-binding method, the interaction could be further identified to quantitatively describe the effect of the interaction between $p_{z}$ and $p_{y}$ orbitals on the negative Poisson's ratio (Li et al. (2021)).

In addition, we also find that when the VP possesses a negative Poisson's ratio, the conduction band and the valence band gradually overlap with each other as the strain increases, which leads to the disappearance of the band gap and thus the semiconductorto-metal transition as shown in Fig. 8(d). Therefore, VP is a meta-material that possesses both negative Poisson's ratio and semiconductor-to-metal transition when under tension. The band gaps of monolayer, bilayer, bulk VP without strain are predicted to be $1.50 \mathrm{eV}$, $1.39 \mathrm{eV}$, and $1.05 \mathrm{eV}$, respectively. The critical uniaxial strain of semiconductor-to-metal transition for monolayer, bilayer, and bulk VP are $0.44,0.32$, and 0.34 , respectively. The semiconductor-to-metal transition usually results in significant changes in physical properties such as resistivity, magnetism, optical reflectivity, etc (Kim et al. (2018)). These unique physical properties together with the negative Poisson's ratio makes VP have broad application prospects in the fields of piezoelectricity, information storage, sensors, field effect transistors and so on.

\section{Conclusion}

In summary, DFT calculations are performed to comprehensively investigate the mechanical responses of monolayer, bilayer and bulk VP under uniaxial tension. In addition, the mechanical properties including tensile strength, fracture strain, Young's modulus, and Poisson's ratio of VP structures are examined. Our results show a unique phase transformation from op structure to cp structure occurring in the VP, which is induced by the change of deformation mechanisms of VP during the loading process. The effect of phase transformation on the elastic properties of VP structures are investigated in details. A very strong anisotropy of elastic properties including Young's modulus, shear modulus, and Poisson's ratio are found in both op and cp phases of VP structures. Specifically, we find that both op and cp phases of all VP structures possess a zero Poisson's ratio in a specific in-plane angle. Moreover, an extremely large in-plane Poisson's ratio and a negative out-ofplane Poisson's ratio are found in the VP structures subject to certain strain. In addition, a semiconductor-to-metal transition phenomenon is observed in all VP strictures under tensile loading. 


\section{Credit author contribution statement}

Penghua Ying: Conceptualization, Methodology, Software, Formal analysis, Investigation, Writing - Original Draft. Writing - Review \& Editing. Xiaowen Li: Methodology, Software, Formal analysis, Writing - Review \& Editing. Xiaobin Qiang: Software, Formal analysis. Yao Du: Software, Formal analysis. Jin Zhang: Methodology, Formal analysis, Writing - Original Draft, Writing - Review \& Editing. Lang Chen: Supervision, Funding acquisition, Writing - Review \& Editing. Zheng Zhong: Supervision, Funding acquisition, Writing - Review \& Editing.

\section{Declaration of competing interest}

The authors declare that they have no known competing financial interests or personal relationships that could have appeared to influence the work reported in this paper.

\section{Acknowledgments}

Z. Zhong acknowledges support from the National Key R\&D Program of China (no. 2018YFB1502602) and the National Natural Science Foundation of China (nos. 11932005 and 11772106). L. Chen acknowledges support from the National Natural Science Foundation of China (51972160) and the Science and Technology Research Items of Shenzhen (JCYJ20180504165650580). P. Ying and J. Zhang thank Huarui Sun, Ligang Sun, and Bo Zou for helpful discussions and insightful comments.

\section{References}

Akinwande, D., Brennan, C.J., Bunch, J.S., Egberts, P., Felts, J.R., Gao, H., Huang, R., Kim, J.S., Li, T., Li, Y., et al., 2017. A review on mechanics and mechanical properties of $2 \mathrm{~d}$ materials-graphene and beyond. Extreme Mechanics Letters 13, 42-77.

Bertolazzi, S., Brivio, J., Kis, A., 2011. Stretching and breaking of ultrathin mos2. ACS Nano 5, 9703-9709.

Born, M., 1940. On the stability of crystal lattices. i, in: Mathematical Proceedings of the Cambridge Philosophical Society, Cambridge University Press. pp. 160-172.

Carvalho, A., Wang, M., Zhu, X., Rodin, A.S., Su, H., Neto, A.H.C., 2016. Phosphorene: from theory to applications. Nature Reviews Materials 1, 1-16.

Dai, Z., Liu, L., Zhang, Z., 2019. Strain engineering of 2d materials: issues and opportunities at the interface. Advanced Materials 31, 1805417.

Evans, K.E., Alderson, A., 2000. Auxetic materials: functional materials and structures from lateral thinking! Advanced Materials 12, 617-628.

Fali, A., Snure, M., Abate, Y., 2021. Violet phosphorus surface chemical degradation in comparison to black phosphorus. Applied Physics Letters 118, 163105.

Geim, A.K., 2009. Graphene: status and prospects. Science 324, 1530-1534.

Jasiukiewicz, C., Paszkiewicz, T., Wolski, S., 2008. Auxetic properties and anisotropy of elastic material constants of $2 \mathrm{~d}$ crystalline media. physica status solidi (b) 245, 562-569.

Jasiukiewicz, C., Paszkiewicz, T., Wolski, S., 2010. Auxetic properties and anisotropy of elastic material constants of $2 \mathrm{~d}$ crystalline media [phys. status solidi b 245, no. 3, 562-569 (2008)]. physica status solidi (b) $247,1247-1247$. 
Jiang, J.W., Chang, T., Guo, X., Park, H.S., 2016. Intrinsic negative poissons ratio for single-layer graphene. Nano Letters 16, 5286-5290.

Jiang, J.W., Park, H.S., 2014a. Mechanical properties of single-layer black phosphorus. Journal of Physics D: Applied Physics 47, 385304.

Jiang, J.W., Park, H.S., 2014b. Negative poissons ratio in single-layer black phosphorus. Nature Communications 5, 1-7.

Kim, S.Y., Lee, M.C., Han, G., Kratochvilova, M., Yun, S., Moon, S.J., Sohn, C., Park, J.G., Kim, C., Noh, T.W., 2018. Spectroscopic studies on the metal-insulator transition mechanism in correlated materials. Advanced Materials 30, 1704777.

Kresse, G., Furthmüller, J., 1996. Efficient iterative schemes for ab initio total-energy calculations using a plane-wave basis set. Physical Review B 54, 11169.

Lee, C., Wei, X., Kysar, J.W., Hone, J., 2008. Measurement of the elastic properties and intrinsic strength of monolayer graphene. Science $321,385-388$.

Li, X., Huang, C., Hu, S., Deng, B., Chen, Z., Han, W., Chen, L., 2020. Negative and near-zero poisson's ratios in $2 \mathrm{~d}$ graphene/mos 2 and graphene/h-bn heterostructures. Journal of Materials Chemistry C 8, 4021-4029.

Li, X., Qiang, X., Gong, Z., Zhang, Y., Gong, P., Chen, L., 2021. Tunable negative poissons ratio in van der waals superlattice. Research 2021.

Lu, Y., Guo, J., 2010. Band gap of strained graphene nanoribbons. Nano Research 3, 189-199.

Maździarz, M., 2019. Comment on the computational 2d materials database: high-throughput modeling and discovery of atomically thin crystals. 2D Materials 6, 048001.

Pang, Z., Li, T., 2021. Mechanics and strain engineering of bulk and monolayer bi2o2se. Journal of the Mechanics and Physics of Solids 157, 104626.

Peng, H., Yang, Z.H., Perdew, J.P., Sun, J., 2016. Versatile van der waals density functional based on a meta-generalized gradient approximation. Physical Review X 6, 041005.

Rothenburg, L., Berlin, A., Bathurst, R.J., et al., 1991. Microstructure of isotropic materials with negative poisson's ratio. Nature 354, 470-472.

Scalise, E., Houssa, M., Pourtois, G., Afanasev, V., Stesmans, A., 2012. Strain-induced semiconductor to metal transition in the two-dimensional honeycomb structure of mos 2. Nano Research 5, 43-48.

Sha, Z.D., Pei, Q.X., Ding, Z., Jiang, J.W., Zhang, Y.W., 2015. Mechanical properties and fracture behavior of single-layer phosphorene at finite temperatures. Journal of Physics D: Applied Physics 48, 395303.

Stukowski, A., 2009. Visualization and analysis of atomistic simulation data with ovito-the open visualization tool. Modelling and simulation in materials science and engineering 18, 015012.

Tran, V., Soklaski, R., Liang, Y., Yang, L., 2014. Layer-controlled band gap and anisotropic excitons in few-layer black phosphorus. Physical Review B 89, 235319.

Voigt, W., 1928. Lehrbuch der Kristallphysik. Teubner Verlag, Leipzig.

Wang, V., Xu, N., Liu, J.C., Tang, G., Geng, W.T., 2021. Vaspkit: a user-friendly interface facilitating high-throughput computing and analysis using vasp code. Computer Physics Communications , 108033.

Wei, Q., Peng, X., 2014. Superior mechanical flexibility of phosphorene and few-layer black phosphorus. Applied Physics Letters 104, 251915.

Woo, S., Park, H.C., Son, Y.W., 2016. Poisson's ratio in layered two-dimensional crystals. Physical Review B 93, 075420.

Wu, J., Wang, B., Wei, Y., Yang, R., Dresselhaus, M., 2013. Mechanics and mechanically tunable band gap in single-layer hexagonal boron-nitride. Materials Research Letters 1, 200-206.

Zhang, B., Zhang, L., Wang, Z., Li, Y., Cheng, Y., Ma, L., Zhang, J., 2021. Cross structured two-dimensional violet phosphorene with extremely high deformation resistance. Journal of Materials Chemistry A 9, $13855-13860$.

Zhang, B., Zhang, L., Yang, N., Zhao, X., Chen, C., Cheng, Y., Rasheed, I., Ma, L., Zhang, J., 2022. 2d young's modulus of black phosphorene with different layers. The Journal of Physical Chemistry C 126 , 1094-1098.

Zhang, L., Gu, M., Li, L., Zhao, X., Fu, C., Liu, T., Xu, X., Cheng, Y., Zhang, J., 2020a. High yield 
synthesis of violet phosphorus crystals. Chemistry of Materials 32, 7363-7369.

Zhang, L., Huang, H., Zhang, B., Gu, M., Zhao, D., Zhao, X., Li, L., Zhou, J., Wu, K., Cheng, Y., Zhang, J., 2020b. Csd 1935087: Experimental crystal structure determination.

Zhang, L., Huang, H., Zhang, B., Gu, M., Zhao, D., Zhao, X., Li, L., Zhou, J., Wu, K., Cheng, Y., et al., 2020c. Structure and properties of violet phosphorus and its phosphorene exfoliation. Angewandte Chemie 132, 1090-1096. 